\title{
Farm management extension in a modernizing agriculture
}

\author{
John W. Mellor
}

Department of Agricultural Economics, Cornell University, Ithaca, New York, USA

Received 21 March, 1968

\section{The Dynamics of modernization}

A modernizing agriculture is by definition a highly dynamic agriculture. A continuous stream of new technologies constantly confronts the cultivator with new alternatives in regard to technique and new conditions for choice of input level. In addition price relationships begin to change much more substantially providing constantly changing conditions for choosing the set of crops and livestock products to be produced. The particular set of new technologies available at any given point of time will change the relative cost of production of various crops. This then creates its own dynamic of shifting cropping pattern and consequently changing price relationships, calling for further changes in farming even beyond the area of impact of new technologies. The dynamic of changing cost relationships is strengthened by changes in relative demand which accompany rising incomes. The dynamics of change in agriculture itself contribute to this growth in income both directly through their impact on cultivator incomes and indirectly by facilitating more rapid growth of the nonagricultural part of the economy. Growth in income imposed on a system of widely varying income elasticities increases demand substantially more for some commodities than others. In a low income country the absolute differences in income elasticities amongst various agricultural products is likely to be very large, resulting in widely disparate rates of growth in demand. Cultivators attuned to a dynamic environment will adjust production as relative profitabilities change and thereby minimize the extent of the change in relative prices.

An example of these dynamics at play in India is the recent development of high yielding varieties of wheat and maize with respective maturities such that they can be grown in successive seasons in the same year. Each of these two innovations alone presented farmers with complex choices regarding these technologies with their higher yields, higher input requirements, greater financing needs and problems of scheduling labor. In addition in some areas these two advances to-gether provided a major decrease in the cost of production of maize and wheat relative to sugarcane and a consequent shift of resources from sugarcane to a maize wheat rotation. However in an environment of rising incomes the demand for sugar is growing much more rapidly than the demand for maize and wheat. Thus one should expect from the above action a relative rise in the price of sugar relative to maize and wheat. This might call for a swing back to sugarcane in the area which made the original swing away from sugarcane. However the more likely optimal response would be for other areas

* Paper presented to the conference for Farm Management Specialists of IADP Districts, sponsored by the IADP section of the Directorate of Extension, Hyderabad, India, January 29-30, 1968. 
in which the new maize and wheat technologies were not appropriate to swing resources into sugarcane and away from something else. Thus the impact of the original technology may have a very widespread effect. These dynamics may of course, be imposed on other dynamics including those of public policy, as has been the case for sugar, thereby greatly exaggerating price swings and making the decision making environment even more dynamic.

Rapid change from a situation of relatively static technology and price relationships to one which is highly dynamic places great pressure on the decision making ability of cultivators. On the one hand the need for dynamic decision making increases. On the other hand the preceding relatively static environment has provided cultivators with little experience with decision making in a dynamic environment. It should of course, not be assumed that because cultivators are in reasonably close adjustment to a relatively static environment that they will quickly adjust to a rapidly changing environment. It is not a question of the desire of cultivators to farm efficiently and profitability. The question is to what extent do they have experience in appraising rapidly changing input output and price relationships, modifying new practices to suit their particular conditions and arriving at optimum decisions concerning change. It would not be surprising if cultivators were ill-prepared for the burdens of rapidly changing technology and price relationships. It is this situation which places a heavy burden on farm management extension.

\section{Cultivators' requirements for farm management information}

Cultivators need (a) recommendations as to the new practices, input levels and cropping patterns which are most likely to be profitable to them, (b) information concerning the underlying input-output and price relationships so that they may modify recommendations to suit their own specific conditions and (c) help in learning how to weigh technical information regarding input output ratios and prices and to combine it into a judgement concerning an optimal set of actions for their conditions. The latter is a necessary part of the process because of the wide variation in conditions from farm to farm calling for modification of recommendations. These inter farm conditions differ not only in regard to physical factors such as soils and irrigation facilities but also with resource availabilities as size of holding, family labor force and incomes vary and with contrasting farmer objectives and values each influence calling for different weighting of the costs and returns from various actions.

Two basic types of studies are needed to facilitate farmer decision making. On the one hand synthetic studies are needed (a) to give a rough idea of the efficiency of particular recommendations before they have been widely adopted (b) to help the leader in innovation see the implications of new recommendations, and (c) to guide initial policy for input supply and other aspects of the environment beyond the control of cultivators. On the other hand there is need for systematic study of farmer experience with new technologies and new price relationships. The latter is necessary to provide (a) revised coefficients for synthetic analysis, (b) convincing evidence to farmers who have not yet taken up the innovations and (c) policy information concerning inhibitors to change which are beyond the control of individual cultivators. 


\section{Synthetic studies}

Synthetic studies may be no more than a partial budget showing the cost of a single input and the expected added output from it, and based on experimental data perhaps modified to suit somewhat different conditions of cultivators. At the other extreme a synthetic study may take into account a series of input output and price changes and deal with the total ramifications of these throughout a farm business, suggesting not only changes in practices but changes in cropping patterns and even in basic resource structure. The techniques range from simple arithmatic calculations to the use of complex programing models. A highly complex approach may be subject to increasing error as the very complexity increases the probabilities that noncompensating errors of ommission and commission have been made. More important the very complexity makes it difficult for the experienced extension agent or farmer to understand the assumptions and to spot aspects not suited to their particular conditions. However, whatever the limitations, there is of course no substitute for the synthetic study prior to significant change of the type to be studied on the part of cultivators. In this early stage such study can contribute substantially to the efficiency of decision making. Full use of previous studies of farmer experience and care to provide adaptation to cultivators' actual resource situation and input output relationships can greatly strengthen such studies. A free flow of information back and forth between those making synthetic studies, extension agents and cultivators themselves should increase our capability for such studies. In addition we should recognize the imperfect state of our knowledge and prepare many studies which test stability in regard to the various factors about which we are uncertain. Such tests can often show that assumptions regarding price relationships or input-output relationships within a known reasonable range will not affect the recommendation significantly. Such knowledge is clearly of great help in giving confidence to recommendations.

\section{Studies of farmers experience}

The complexity of farming and the variability in farming conditions require constant checking of synthetic studies against actual cultivator experience. In the case of innovations which require substantial inputs and provide only modest returns it is probably not advisable for the lower income cultivators to be among the earlier innovators. Once a few cultivators have tried an innovation, their experience can be studied in detail and the results of that study made available for guiding the remaining cultivators. Cultivators are more likely and perhaps rightfully so, to have more confidence in such studies than purely synthetic approaches. Particularly while they are inexperienced with measuring input output relationships within a complex environment, cultivators may be ill equipped to make such estimates on their own. Hence studies of this type may greatly increase the rate of spread of innovation. Shortening the spread of innovation from six years to three years will of course, double the rate of growth, a path towards progress not to be taken lightly, particularly if it is backed by a constant stream of new innovations from the experiment stations.

This type of extension information has special importance to lower income cultivators and hence may have special importance in attempts to reduce income disparities. It is likely to be the lower income cultivators who have most to lose by early innovation. Hence study of cultivator experience may have its greatest impact 
in moving knowledge from those best equipped to take risks down to those who most need the benefits of profitable innovations.

\section{Policy implications of extensions oriented farm management research}

Because our knowledge of the total process is so scant, agricultural development policy is necessarily an iterative process in which each successive new development brings to light new program needs. In such a process the key to growth is close contact with cultivators and cultivator conditions so that as each new policy creates its new needs they may be quickly diagnosed and attended. Synthetic studies showing the probable effects of price and technological changes can be very helpful in suggesting new policy needs. Study of farmer experience can serve to confirm or modify these hypotheses and give an indication of the urgency of action.

A particularly important area of such policy influence is in regard to research priorities. Farm management studies show why certain types of innovations are not profitable to cultivators under existing conditions and thereby suggest productive new lines of research. In addition, farm management research may show what types of resources are being underutilized and the bottlenecks which cause such underutilization thereby suggesting lines of research for changing length and time of growing season and other aspects of plant behavior so as to foster increased production through fuller use of existing resources. One of the most important reasons for failure of biological science research to provide the technological basis for agricultural development is a failure to recognize the problems which cultivators face and to provide innovations which solve those problems.

Thus it is important that farm management extension research be oriented and articulated not only forward to the cultivator but also back to the research worker and to the formulators of public and private sector policy with regard to agriculture. 